\title{
Chronic TCDD exposure results in the dysregulation of gene expression in splenic B-lymphocytes and in the impairments in T-cell and B-cell differentiation in mouse model
}

\author{
Yu Feng ${ }^{1}$, Jijing Tian ${ }^{1}$, Irina Krylova ${ }^{2}$ Tuan Xu ${ }^{1}$, Heidi Qunhui Xie ${ }^{1}$, Tai L. Guo ${ }^{3}$, Bin Zhao ${ }^{1, *}$ \\ 1. Research Center for Eco-Environmental Sciences, Chinese Academy of Sciences, Beijing 100085, China. E-mail: fyuivy@163.com \\ 2. XCellAssay, LLC, San Francisco, CA, 94107, USA \\ 3. Department of Veterinary Biosciences and Diagnostic Imaging, College of Veterinary Medicine, University of Georgia, Athens, GA 30602-7382, USA
}

\section{A R T I C L E I N F O}

\section{Article history:}

Received 31 July 2015

Revised 1 November 2015

Accepted 2 November 2015

Available online 2 January 2016

\section{Keywords:}

Chronic

TCDD

B cell

transcriptome

EphA2

CAMP

\begin{abstract}
A B S T R A C T
2,3,7,8-Tetrachlorodibenzo-p-dioxin (TCDD) exposure in humans is associated with marked immune suppressions and increased incidence of lymphoblastic diseases. To elucidate mechanisms of impairments in humoral immune responses, we used a murine model. Following a 20-week administration of low doses of TCDD, we observed severely reduced antibody titers, dramatically decreased number of splenic Th1 and Th2 cells and an increase in $\mathrm{CD} 19^{+} \mathrm{B}$ cells. Transcriptional profiling of $\mathrm{CD} 19^{+} \mathrm{B}$ cells showed that markers of pre-B cells were significantly elevated, indicating delayed B cell maturation. These changes in B cells were accompanied by decreases of $\mathrm{T}$ helper cell numbers and reduced IgM and IgG titers. A transcriptome analysis of splenic B cells followed by Ingenuity Pathway Analysis (IPA) revealed a set of differentially expressed genes known to play roles in tumorigenesis, cell-proliferation and cell-migration. The most up-regulated transcript gene was Eph receptor A2 (EphA2), a known oncogene, and the most down-regulated transcript was ZBTB16 that codes for a negative transcriptional regulator important in epigenetic chromatin remodeling. IPA identified cAMP-responsive element modulator (CREM) and cAMP-responsive element binding protein 1 (CREB1) as top upstream regulators. Consistently, a MAPPER promoter database analysis showed that all top dysregulated genes had CREM and/or CREB1 binding sites in their promoter regions. In summary, our data showed that chronic TCDD exposure in mice caused suppressed humoral immunity accompanied with profound dysregulation of gene expression in splenic B-lymphocytes, likely through cAMP-dependent pathways. This dysregulation resulted in impairments in T-cell and B-cell differentiation and activation of the tumorigenic transcription program.

(C) 2015 The Research Center for Eco-Environmental Sciences, Chinese Academy of Sciences.
\end{abstract}

Published by Elsevier B.V.

\section{Introduction}

2,3,7,8-Tetrachlorodibenzo-p-dioxin (TCDD) is a persistent environmental pollutant that targets various organs including the immune system in both acute and chronic exposure. Even at doses with no obvious signs of toxicity, TCDD alters the immune functions in virtually every species studied so far (Holsapple et al., 1991; Kerkvliet, 2002). TCDD acts via the activation of the aryl hydrocarbon receptor (AhR), a ligand-activated transcription factor that belongs to the basic

\footnotetext{
* Corresponding author. E-mail: binzhao@rcees.ac.cn (Bin Zhao).
} 
helix-loop-helix superfamily whose members play key roles in gene expression networks underlying many essential physiological and developmental processes. Human and animal exposures to TCDD result in immune suppression that is not observed in AhR null mice (Vorderstrasse et al., 2001a). AhR is involved in both innate and acquired immune responses and has been shown to be essential for the differentiation and maintenance of the intestinal intraepithelial lymphocytes (IELs) and the innate lymphoid cells (ILCs) (Li et al., 2011; Spits and Di Santo, 2011). The role of AhR in the Th17 and T regulatory (Treg) cell development is firmly established (Ho and Steinman, 2008). B cells are also highly sensitive targets of TCDD (Sulentic and Kaminski, 2011b). Epidemiologic evidence shows that TCDD exposure depresses humoral immunity in human subjects. As such, both Seveso accident subjects with the highest TCDD exposure and Vietnam Veterans exhibited decreased plasma IgG levels and increased incidence of lymphatic tumors, especially non-Hodgkin's lymphoma (NHL) (Sulentic and Kaminski, 2011b).

TCDD has been demonstrated to suppress the primary IgM response in purified splenic B cells (Dooley and Holsapple, 1988). This suppression, both in vivo and in vitro, involved the activation of AhR (Vorderstrasse et al., 2001b). Another TCDD effect on immune system is a shift in the Th1/Th2 balance, an important measure of the immune system fitness (Kidd, 2003). A large body of data demonstrates the effects of AhR activation on the differentiation of the T-cell subsets (Nguyen et al., 2013), including a number of genome-wide expression studies on T-cells (Li et al., 2013; Stubbington et al., 2015). While the roles of AhR in B cell differentiation are demonstrated (De Abrew et al., 2011; Zhang et al., 2013), there is a dearth of data that allows a better mechanistic understanding of the long-term consequence of the chronic TCDD exposure on humoral immunity (e.g., B cells) that underlie referenced above adverse outcomes of TCDD exposure (Kerkvliet, 2002). With the application of transcriptome analysis, we could gain a full view of the effects of TCDD on B lymphocytes at the transcriptional level. Transcriptomic studies have been performed on B cell lines (De Abrew et al., 2010); however, to our knowledge, there were no such in vivo studies reported regarding $\mathrm{B}$ lymphocytes isolated from mice chronically treated with TCDD. In the current study, we used a murine model that recapitulates a chronic human exposure to TCDD to evaluate the effects of TCDD on the immune phenotype and the splenic B cells transcriptome. Specifically, splenic B lymphocytes were isolated after a 20-week TCDD exposure at an environmentally relevant dose for transcriptomic analysis. Our analysis provides an important insight into the possible mechanisms of the impairments in the humoral immunity and into the increased incidence of lymphatic tumors resulting from the chronic TCDD exposure in humans.

\section{Material and methods}

\subsection{Animals}

Twenty-four male C57BL/6 mice ( 6 weeks old, $20 \pm 1 \mathrm{~g}$ ) were purchased from Vital River Laboratories (VRL; Beijing, China) and housed in the Animal Research Center of Tsinghua University under specific pathogen-free conditions, at a controlled temperature of $24 \pm 2^{\circ} \mathrm{C}$ and humidity of $50 \% \pm$ $10 \%$, with a cycle of $12 \mathrm{hrs}$ light and $12 \mathrm{hrs}$ dark. Animals were provided with pellet foods and water ad libitum, and randomly assigned into three groups, two TCDD treated groups and one vehicle control (Mice received DMSO diluted in olive oil served as the control). TCDD (Toronto Research Chemicals Inc., Canada) was dissolved in DMSO (Sigma-Aldrich, St. Louis, USA) at stock concentrations of $0.1 \mu \mathrm{g} / \mu \mathrm{L}$ and $0.01 \mu \mathrm{g} / \mu \mathrm{L}$, and then diluted 1000 times in olive oil as dosing solutions. The mice in two treated groups were administered with TCDD diluted in olive oil at 2 doses (low: 0.1, high: $1 \mu \mathrm{g} / \mathrm{kg}$ of body weight (bw)) by gavage every other week. Doses used in the current study were based on previous TCDD chronic exposure reports. In a 13-14 month-chronic exposure study, C57BL/6 mice were given $0.2 \mu \mathrm{g} / \mathrm{kg}$ bw TCDD for phenotype analysis of spleen, thymus and peripheral blood (Oughton et al., 1995). Body weights were measured at the time of gavage. After the 20 weeks of exposure, blood samples were collected immediately after euthanasia for serum isolation, and spleen tissues were processed for further studies. All animal experiments were conducted in accordance with animal protocols approved by the Animal Care and Use Committee of Tsinghua University.

\subsection{Flow cytometric analysis}

Spleens were harvested, and single cell suspensions were prepared by homogenizing on $70 \mu \mathrm{m}$ cell strainers (BD Falcon, Bedford, MA, USA) using PBS with $2 \%$ FBS. Spleen red blood cells were removed at $37^{\circ} \mathrm{C}$ by adding $2 \mathrm{~mL}$ of $1 \times$ lysing buffer (BD Biosciences, San Jose, USA) for $3 \mathrm{~min}$. Then, cells were washed twice with cold PBS, and stained for $30 \mathrm{~min}$ in an appropriate diluted antibody staining solution. Antibodies used were from BD Biosciences, including rat anti-mouse CD3-Percp-Су ${ }^{\mathrm{TM}} 5.5$, rat anti-mouse CD4-FITC and rat anti-mouse CD8-PE-Cy ${ }^{\mathrm{TM}} 7$, and isotype controls were Percp-Cy ${ }^{\mathrm{TM}} 5.5$ rat IgG2a, FITC rat IgG2a and PE-Cy ${ }^{\mathrm{TM}} 7$ rat IgG2a, respectively. For each $10^{6}$ cells, $0.5 \mu$ g antibody was used for staining.

For intracellular cytokine staining, $10^{5}$ cells were stimulated with Leukocyte Activation Cocktail, with BD GolgiPlug ${ }^{\mathrm{TM}}$ (BD Biosciences) at $37^{\circ} \mathrm{C}$ in a $5 \% \mathrm{CO}_{2}$ incubator for $5 \mathrm{hrs}$. The stimulated cells were collected and washed twice with PBS, and permeabilized using Perm/Wash ${ }^{\mathrm{TM}}$ buffer for $15 \mathrm{~min}$ at room temperature. After spinning and removing the supernatant, cells were stained with PE anti-mouse IFN- $\gamma$ for Th1 cell subset and PE-Cy' ${ }^{\mathrm{TM}} 7$ anti-mouse IL-4 for Th2 cell subset for $30 \mathrm{~min}$ on ice in the dark. For Treg staining, similar procedures were employed except that $10^{5}$ cells were used for stimulation. The stimulated cells were collected and washed, and then stained with FITC anti-mouse CD4 for 30 min on ice in the dark. After washed twice with cold PBS, cells were incubated in $1 \mathrm{~mL}$ mouse Foxp3 permeabilization buffer (BD Biosciences) for $30 \mathrm{~min}$ at room temperature in the dark. After washing, cells were stained with V450 anti-mouse FoxP3 for 30 min on ice in the dark. Data were collected on BD LSRII (BD Biosciences) and analyzed using CellQuest software.

\subsection{Luminex analysis}

The concentrations of selected cytokines (IL-6, IL-12p70, IL-13, and IL-22) in mouse sera were determined by Luminex assay 
with a custom kit, Mouse Th17 magnetic Bead Panel (EMD Millipore Corporation, Billerica, MA, USA) according to the manufacturer's instructions. The instrument used was Luminex 200TM (Luminex, Austin, TX, USA) and read under Mag Plex. Data were analyzed using Milliplex Analyst 5.1 Software (Merck Millipore). All samples were tested in duplicate for Luminex assay.

\subsection{ELISA}

Levels of serum IgG, IgM and IgA were measured using ELISA kits purchased from eBioscience (eBioscience, San Diego, CA) according to the manufacturer's instructions. Briefly, capture antibodies (1:1000. V/V) were coated separately on microtiter plates, incubated overnight at $4^{\circ} \mathrm{C}$. Capture antibodies used were purified anti-mouse IgG monoclonal antibody, purified anti-mouse IgM monoclonal antibody and purified anti-mouse IgA monoclonal antibody for IgG, IgM and IgA, respectively. After washing with PBS containing 0.05\% Tween-20 (PBST), microplate wells were blocked with 5\% BSA at room temperature for 2 hrs. After washing with PBST, diluted samples were added to the microplate and incubated for $2 \mathrm{hrs}$ at room temperature. After washing with PBST, HRP-conjugated detection antibody was added to each well and incubated for $1 \mathrm{hr}$ at room temperature. HRP-conjugated detection antibodies used were: HRP-conjugated anti-mouse IgG monoclonal antibody, HRP-conjugated anti-mouse $\operatorname{Ig}(\mathrm{H}+\mathrm{L})$ monoclonal antibody and HRP-conjugated anti-mouse IgA monoclonal antibody for IgG, IgM and IgA, respectively. After washing with PBST, TMB was added and incubated for $15 \mathrm{~min}$ at room temperature. The enzyme reaction was stopped with $1 \mathrm{~mol} / \mathrm{L} \mathrm{H}_{3} \mathrm{PO}_{4}$. The optical density was read using a microplate fluorescence reader (Tecan Infinite F200Pro Switzerland) at a wavelength of $450 \mathrm{~nm}$.

\subsection{Transcriptome analysis}

After lysis of the red blood cells, CD19 positive cells were selected from single spleen cell suspension of control and $0.1 \mu \mathrm{g} / \mathrm{kg}$ bw groups using mouse CD19 positive selection kit (StemCell Technologies, Meylan, France) according to the manufacturer's instructions. Total RNA was purified from CD19 positive cells using TRIzol reagent (Invitrogen, Carlsbad, CA). Also, the RNA was sent to perform the transcriptome study.

The original image data were translated into sequence data as raw reads via base calling analysis. After a stringent filtering reads, all clean reads were mapped to the reference genome (available at ftp://ftp.ensembl.org/pub/release-75/ fasta/homo_sa piens/dna/) from the Ensembl database using the Short Oligonucleotide Analysis Package alignment tool SOAP aligner/SOAP2. To identify the differentially expressed genes between control and TCDD treated group, data for each gene were normalized and calculated as RPKM (Reads Per KiloBase per Million of mapped reads) as described by Mortazavi et al. (2008). The differentially expressed genes were subjected to Kyoto Encyclopedia of Genes and Genomes (KEGG) enrichment analysis using DAVID (The Database for Annotation, Visualization and Integrated Discovery) with the total mouse genome information as the background (Ashburner et al., 2000; Dennis Jr et al., 2003; Huang et al., 2009; Kanehisa and Goto, 2000). The data were further analyzed with Ingenuity Pathway Analysis (IPA). In IPA analysis, an upstream regulator analysis is a remarkable step forward in transcription regulator prediction (Krämer et al., 2014). Given the differential regulation of up- and down-regulated genes, the activation states of upstream regulators are determined by the regulation direction associated with relationship of the gene's regulator. In IPA analysis, activation of z-score is used as an independent test to identify the significant upstream regulators.

\subsection{Statistical analysis}

Experimental data were analyzed by one-way ANOVA or Dunnett test using GraphPad Prism 5 (GraphPad Software, USA). Results are presented as mean \pm SEM. Differences were classified as significant ${ }^{*}$ for $p<0.05$ and ${ }^{* *}$ for $p<0.01$ from control.

\section{Results}

\subsection{Body weight showed no difference after TCDD exposure}

Body weights were recorded at the time of gavage. In the $0.1 \mu \mathrm{g} / \mathrm{kg}$ bw treatment group, bw showed a tendency of increase, but this trend was not found in the $1 \mu \mathrm{g} / \mathrm{kg}$ bw treatment group. Through the 20-week exposure, no statistically significant change of bw was found (Fig. 1).

\subsection{Chronic TCDD exposure dramatically reduced the numbers of Th1 and Th2 lymphocytes, shifted the Th1/Th2 ratio, and decreased the levels of pro-inflammatory cytokines IL- 6 and IL-22}

As shown in Table 1 and Fig. 2, chronic TCDD exposure dramatically reduced the percentages of both Th1 and Th2 lymphocytes; however, the Th1/Th2 ratio was increased following TCDD treatment at $1 \mu \mathrm{g} / \mathrm{kg}$ bw treatment group, suggesting the Th1/Th2 balance was shifted toward the Th1 cells. TCDD treatment only weakly affected the number of Tregs, consistent with the literature (Kerkvliet, 2009b; Negishi et al., 2005). We also found that TCDD exposure decreased the levels of pro-inflammatory cytokines IL- 6 and IL-22 and increased the levels of the anti-inflammatory cytokines IL-13 and IL-12p70 (Table 2), consistent with TCDD-induced suppression of immune hypersensitivity phenotype in the mouse model of peanut allergy (Schulz et al., 2011).

\subsection{Chronic TCDD exposure was associated with reduced immunoglobulin titers}

Next, we tested the percentage of splenic CD19+ B lymphocytes and levels of three Igs, to evaluate effects of TCDD on the humoral immunity and B cell function. As shown in Table 3 and Fig. 3, the percentages of CD $19^{+}$B lymphocytes were increased significantly by $8.2 \%$ and $7.9 \%$ in 0.1 and $1 \mu \mathrm{g} / \mathrm{kg}$ bw treatment group, respectively. Serum levels of IgG were decreased significantly in both treatment groups and IgM level was decreased in the $1 \mu \mathrm{g} / \mathrm{kg}$ bw treatment group. IgA levels showed no obvious changes after chronic TCDD treatment. 


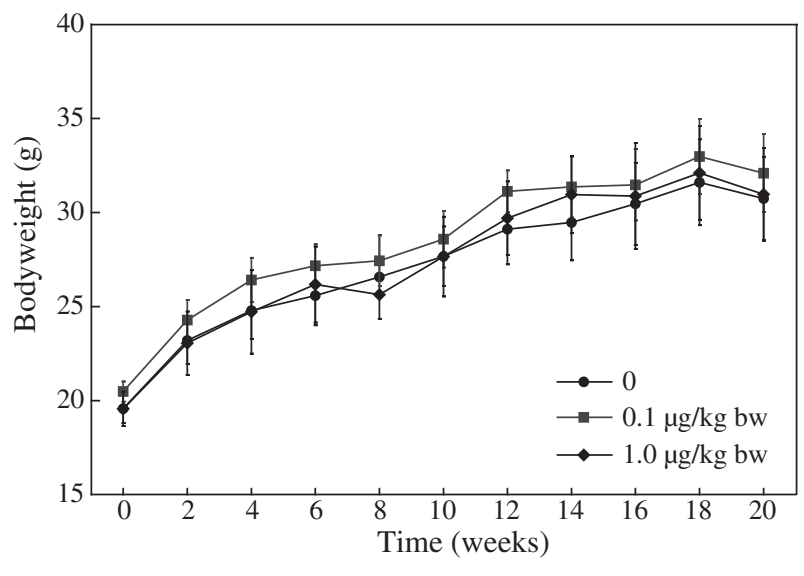

Fig. 1 - Body weights were measured at the time of gavage. No significant changes were found among three groups in the 20-week period of 2,3,7,8-tetrachlorodibenzo-p-dioxin (TCDD) exposure. $n=5-8$.

\subsection{Chronic exposure to TCDD was associated with marked changes in the splenic B cell transcriptome}

To gain the insight into the mechanistic processes that underlie the observed changes in the immune functions, we performed the RNA-seq to identify a gene set that was differentially expressed in mice chronically exposed to TCDD when compared to control. TCDD exposure exerted marked changes on the splenic B cells transcriptome. Using the threshold of the absolute value of the fold change being $\geq 2.0$, the FDR corrected $p$-value being $\leq 0.001$, we identified 70 differentially expressed transcripts. Ten most over-expressed and ten most under-expressed genes are shown in the Table 4. To identify which molecular pathways involved in the observed differential expression, we performed the IPA analysis. Table 5 shows the results of the Downstream Effect Analysis that revealed the most dysregulated pathways by TCDD. Consistent with the increased percentage of B cells, the most up-regulated pathway was that of lymphocyte proliferation (Z-score 2.5), with the specific B cell proliferation pathway also up-regulated (Z-score 1.1). Another set of up-regulated pathways included the pathways for invasion, migration, and movement of cells, consistent with the growing body of evidence that supports the roles of AhR in cell growth, movement and proliferation, as

\section{Table $1-\mathrm{CD}^{+}$cell differentiation and Th1/Th2 ratio.}

\begin{tabular}{|c|c|c|c|c|}
\hline \multirow[t]{2}{*}{ Cell subset } & & \multirow[t]{2}{*}{ Control } & \multicolumn{2}{|c|}{ TCDD $(\mu \mathrm{g} / \mathrm{kg} \mathrm{bw})$} \\
\hline & & & 0.1 & 1 \\
\hline \multirow[t]{2}{*}{ Th1 } & $\%$ Total & $2.33 \pm 0.2$ & $1.73 \pm 0.08^{* *}$ & $1.55 \pm 0.08^{* *}$ \\
\hline & $\%$ Ctrl & 100 & 74 & 67 \\
\hline \multirow[t]{2}{*}{ Th2 } & $\%$ Total & $4.29 \pm 0.68$ & $3.70 \pm 0.68^{*}$ & $1.79 \pm 0.39^{*}$ \\
\hline & \% Ctrl & 100 & 86 & 42 \\
\hline \multirow[t]{2}{*}{ Treg } & $\%$ Total & $2.58 \pm 0.25$ & $2.09 \pm 0.08$ & $2.07 \pm 0.06$ \\
\hline & $\%$ Ctrl & 100 & 81 & 80 \\
\hline Th1/Th2 & & 0.54 & 0.47 & 0.86 \\
\hline
\end{tabular}

${ }^{*} p<0.05$ and ${ }^{* *} p<0.01$, data represents mean \pm SEM, $n=5-8$. well as in the regulation of TGF- $\beta$ activity (Carvajal-Gonzalez et al., 2009; Fernandez-Salguero, 2010; Gomez-Duran et al., 2009; Kerkvliet, 2009a; Santiago-Josefat et al., 2004). The pathway regulating the apoptosis of cancer cells was the most down-regulated, consistent with the established lymphomagenic potential of TCDD.

2.5. The top upstream regulatory networks and the top upstream regulators identified by the Ingenuity Pathway Analysis

The analysis of the top upstream regulatory networks has identified the top downstream "diseases and functions" as vasculogenesis (consistency score 7.43) and binding of DNA (consistency score -6.0). Significant positive consistency score for vasculogenesis suggested that differentially expressed genes were involved in positive regulation of vasculogenesis that was consistent with known effects of AhR in cardiovascular physiology and disease (Lund et al., 2003). Significant negative consistency score for the "binding of DNA" module indicated that many of the differentially expressed genes were involved in gene transcription (Table 5). IPA Molecular and Cellular analysis module indicated that 31 (44\% of total) differentially expressed molecules were involved in gene expression, while 39 molecules (55\% of total) were involved in cellular development, growth and proliferation (Appendix A Table S1).

Top upstream transcriptional regulators identified by the IPA Core Analysis were CREM and CREB1. We used a Mapper database (Marinescu et al., 2005a), online link http://genome. ufl.edu/mapper/, to analyze $10 \mathrm{~kb}$ regions upstream of the ATG codon in the top 10 up-regulated and top 10 down-regulated molecules in the dataset (Table 6). Our analysis showed that CREM and/or CREB1 binding sites were present in all promoters but one of the analyzed genes. Interestingly, only the top up-regulated genes contained dioxin-response elements (DREs) in their promoters, whereas all top down-regulated genes lacked DREs. Other members of the cAMP-dependent signaling pathway overexpressed in our dataset included the AP1 transcription factors FosL2 (Log2 = 2.26) and JunB (Log2 = 1.03). Cyclic AMP-dependent transcription factor, ATF3, was the third most up-regulated transcript in our dataset (Log2, 2.88). Using Mapper analysis, we found one DRE, two CREM, two CREB1, and two ATF3 binding sites in the $10 \mathrm{~kb}$ promoter region preceding the ATG of mouse ATF3 gene, suggesting that TCDD exposure can induce a positive feedback regulation of the ATF3 transcript, potentially leading to the up-regulation of the ATF3 target genes. Taken together, these results demonstrated that chronic TCDD exposure resulted in marked and durable changes in splenic B-cell transcription program.

2.6. Chronic TCDD exposure affected mitogen-activated protein kinase signaling pathway, circadian rhythm genes and non-Hodgkin's lymphoma-associated genes

In addition to the IPA analysis, we performed a KEGG pathway analysis and identified that mitogen-activated protein kinase (MAPK) signaling pathway and circadian rhythm pathway were dysregulated following chronic TCDD exposure. Table 7 

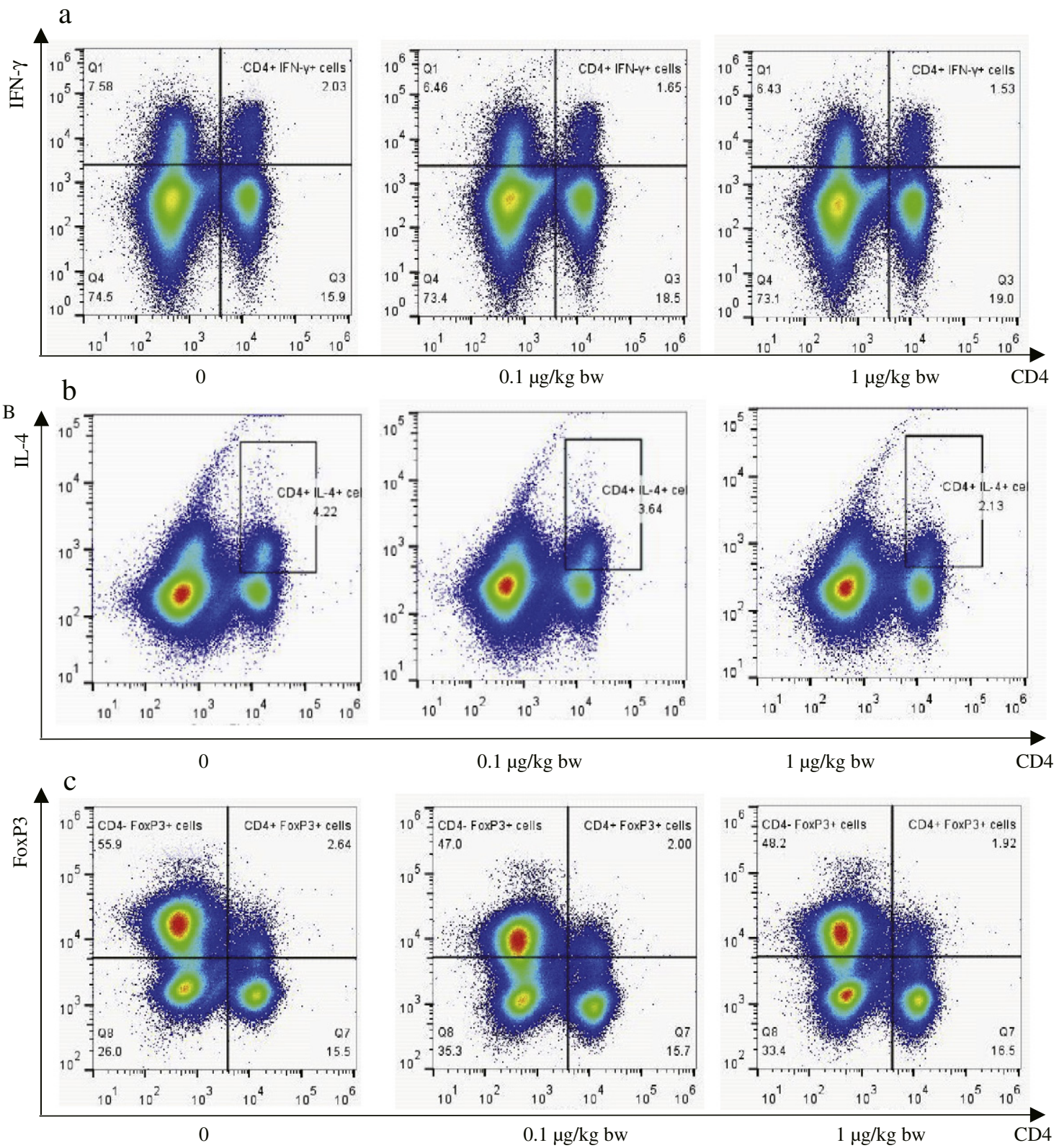

Fig. 2 - Effects of 2,3,7,8-tetrachlorodibenzo-p-dioxin (TCDD) on T cell development in spleen. (a) Representative dot plots of Th1 cell subset; (b) representative dot plots of Th2 cell subset; (c) representative dot plots of Treg cell subset. $n=5-8$.

shows the differentially expressed genes involved in these two pathways.

Since the accidence of the non-Hodgkin's lymphoma (NHL) is significantly increased in the cases of human TCDD exposure as mentioned before, we compared our current data set of differentially expressed genes with the reported NHL-associated genes data sets, as well as with the IPA NHL-associated genes (Lan et al., 2015a). Fig. 4 shows that

Table 2 - Cytokine changing pattern after chronic TCDD exposure.

\begin{tabular}{llccc} 
& Cytokine $(\mathrm{pg} / \mathrm{mL})$ & 0 & $0.1 \mu \mathrm{g} / \mathrm{kg}$ bw & $1 \mu \mathrm{g} / \mathrm{kg}$ bw \\
\hline Pro-inflammation & IL-6 & $7.13 \pm 2.02$ & $5.03 \pm 1.2$ & $3.85 \pm 0.71$ \\
& IL-22 & $14.53 \pm 3.87$ & $16.48 \pm 4.75$ & $8.74 \pm 1.82$ \\
Anti-inflammation & IL-13 & $261.98 \pm 36.24$ & $209.60 \pm 35.26$ & $305.84 \pm 43.81$ \\
& IL-12p70 & $15.75 \pm 3.63$ & $14.56 \pm 2.9$ & $17.14 \pm 4.12$ \\
\hline Data represents mean \pm SEM, $n=$ 5-8. & & & \\
\hline
\end{tabular}


Table 3-CD19+ B cell proportion and serum antibody levels.

\begin{tabular}{lrrr} 
& \multicolumn{1}{c}{0} & $0.1 \mu \mathrm{g} / \mathrm{kg}$ bw & $1 \mu \mathrm{g} / \mathrm{kg} \mathrm{bw}$ \\
\hline CD19+ B cell $(\%)$ & $38.84 \pm 2.93$ & $47.04 \pm 1.72^{*}$ & $46.71 \pm 1.76^{*}$ \\
IgM $(\mathrm{pg} / \mathrm{mL})$ & $3.90 \pm 0.15$ & $2.75 \pm 0.26^{*}$ & $2.65 \pm 0.31^{* *}$ \\
$\left(\times 10^{4}\right)$ & & & \\
IgG $(\mathrm{pg} / \mathrm{mL})$ & $74.41 \pm 21.81$ & $55.32 \pm 20.53$ & $12.74 \pm 1.28^{*}$ \\
$\left(\times 10^{4}\right)$ & & & \\
IgA $(\mathrm{pg} / \mathrm{mL})$ & $7.73 \pm 2.82$ & $3.84 \pm 1$ & $6.60 \pm 1.83$ \\
$\left(\times 10^{4}\right)$ & & & \\
\hline${ }^{*} p<0.05$ and ${ }^{* *} p<0.01$, data represents mean $\pm \mathrm{SEM}, n=5-8$.
\end{tabular}

four of the NHL-associated genes, IRF4, Tgif1, Fam46c and Dusp4, were present in our data sets.

\section{Discussion}

Many effects of TCDD have been studied following an acute exposure in experimental animals. In contrast, humans are exposed to the low daily doses of these chemicals inadvertently present in the environment (DeVito et al., 1995). In the current study, we used a murine model to mimic the chronic exposure of TCDD. We found dramatic changes of both humoral and cellular components of the immune system in exposed mice. After the 20-week exposure, percentages of both Th1 and Th2 subsets were decreased and cytokine patterns were changed as pro-inflammatory cytokines of IL-6 and IL-22 were decreased while anti-inflammatory cytokines of IL-13 and IL-12p70 were increased. These results, together with the significant decreases in IgG and IgM titers, indicated that the immune responses were suppressed. These results were consistent with previous reports following human accident exposures (Sulentic and Kaminski, 2011b), indicating that the selected murine model accurately recapitulated a paradigm of the chronic human exposure of TCDD and could provide information for further studies.

One of the reasons leading to dramatically reduced IgG and IgM production could be the decrease in number of the antibody-producing plasma cells, due to the arrest in the $\mathrm{B}$
Table 4-Top ten up-regulated and down-regulated genes.

\begin{tabular}{llcl} 
Gene symbol & Gene ID & Log2 ratio & $p$-Value \\
\hline Epha2 & 010139 & 10.40637526 & $6.61 \mathrm{E}-06$ \\
MAFF & 010755 & 2.922906966 & $6.90 \mathrm{E}-10$ \\
ATF3 & 007498 & 2.882902008 & $1.03 \mathrm{E}-13$ \\
Eps8l1 & 026146 & 2.825738307 & $9.13 \mathrm{E}-08$ \\
Irs2 & 001081212 & 2.808303844 & $1.25 \mathrm{E}-12$ \\
Osgin1 & 027950 & 2.749424145 & $6.12 \mathrm{E}-07$ \\
Nr4a1 & 010444 & 2.313201751 & $5.20 \mathrm{E}-12$ \\
Fosl2 & 008037 & 2.256546043 & $8.08 \mathrm{E}-13$ \\
Zbtb10 & 77660 & 2.23845681 & $1.34 \mathrm{E}-13$ \\
B3gnt7 & 145222 & 2.152388812 & $3.44 \mathrm{E}-13$ \\
Zbtb16 & 001033324 & -9.680039889 & $1.85 \mathrm{E}-06$ \\
Hspa1l & 013558 & -5.022536871 & $1.13 \mathrm{E}-07$ \\
Erdr1 & 133362 & -2.942128787 & $6.67 \mathrm{E}-51$ \\
Recql & 001204906 & -1.712681608 & $9.05 \mathrm{E}-06$ \\
Serpine2 & 009255 & -1.608845797 & $7.66 \mathrm{E}-09$ \\
Zbtb18 & 013915 & -1.469410457 & $1.90 \mathrm{E}-05$ \\
Zfp455 & 001048204 & -1.458635986 & $8.65 \mathrm{E}-06$ \\
Tmpo & 001080134 & -1.41569987 & $7.01 \mathrm{E}-07$ \\
Zfp759 & 172392 & -1.390753513 & $1.19 \mathrm{E}-05$ \\
Zfp948 & 001002008 & -1.382533006 & $1.11 \mathrm{E}-06$ \\
\hline
\end{tabular}

cell maturation. B cell differentiation into the plasma cell is predominantly triggered by the contact with the $\mathrm{T}$ helper cells. The Th2 cells stimulate the immature B cells to proliferate into the antibody secreting plasma cells while Th1 cells promote the production of IgG (Licona-Limón et al., 2013; MacLeod et al., 2011; Smith et al., 2000). The TCDD exposure has led to a dramatic reduction in both Th1 and Th2 cells; that alone is expected to cause a partial arrest in B cell maturation into plasma cells. While we observed moderate increase in $\mathrm{CD}^{+} 9^{+}$cells, these cells exhibited a genetic signature of the immature B cells characterized by significantly increased expression of the pre-B markers, transcriptional factors Gfi1 and IRF4 (Ellmeier and Taniuchi, 2014). Thus, the splenic B-cell phenotype was characterized by the accumulation of the pre-B cells, ultimately leading to the reduction of antibody secretion following chronic TCDD exposure.

The results of the international cohort study that examined workers exposed to dioxin showed a strong positive
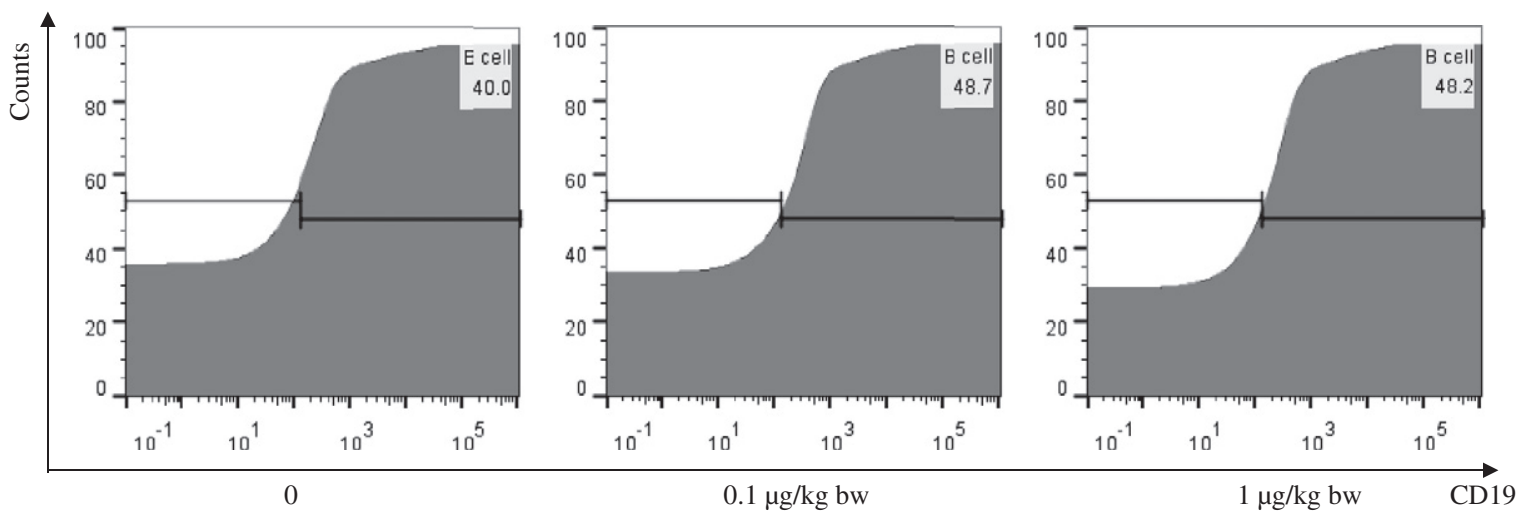

Fig. 3 - Effects of TCDD on B cell development (percentage of CD19+ B cell) in spleen. $n=5-8$. 


\begin{tabular}{|c|c|c|}
\hline $\begin{array}{l}\text { Downstream } \\
\text { effects }\end{array}$ & z-score & Genes involved \\
\hline $\begin{array}{l}\text { Proliferation of } \\
\text { lymphocytes }\end{array}$ & 2.533 & $\begin{array}{l}\text { BHLHE41 ADRB2 EHPA2 PIM3 AHNAK } \\
\text { IRS2 GR1 CCR7 IRF4 BHLHE40 ZBTB16 } \\
\text { CD274 JUNB }\end{array}$ \\
\hline Invasion of cells & 1.908 & $\begin{array}{l}\text { EPHA2 ATF3 IRS2 NR4A1 BHLHE40 CCR7 } \\
\text { CD274 TP53INP2 KDM6B PLAUR AHNAK } \\
\text { JUNB ADRB2 SERPINE2 ZBTB16 }\end{array}$ \\
\hline Cell movement & 1.537 & $\begin{array}{l}\text { EPHA2 ATF3 IRS2 OSGIN1 NR4A1 FOSL2 } \\
\text { BHLHE40 CCR7 CD274 LITAF TP53INP2 } \\
\text { KDM6B DUSP4 PLAUR AHNAK TRIB1 } \\
\text { ADRB2 DDIT4 LIFR ZBTB18 SERPINE2 } \\
\text { Erdr1 ZBTB16 }\end{array}$ \\
\hline Migration of cells & 1.530 & $\begin{array}{l}\text { EPHA2 ATF3 IRS2 OSGIN1 NR4A1 } \\
\text { BHLHE40 CCR7 CD274 LITAF TP53INP2 } \\
\text { KDM6B DUSP4 PLAUR AHNAK ADRB2 } \\
\text { DDIT4 ZBTB18 SERPINE2 Erdr1 ZBTB16 }\end{array}$ \\
\hline $\begin{array}{l}\text { Invasion of } \\
\text { tumor cell lines }\end{array}$ & 1.387 & $\begin{array}{l}\text { EPHA2 ATF3 IRS2 NR4A1 CCR7 } \\
\text { TP53INP2 KDM6B PLAUR JUNB ADRB2 } \\
\text { SERPINE2 }\end{array}$ \\
\hline $\begin{array}{l}\text { Proliferation of B } \\
\text { lymphocytes }\end{array}$ & 1.117 & IRS2 IRF4 JUNB PIM3 ZBTB16 \\
\hline $\begin{array}{l}\text { Differentiation } \\
\text { of myeloid cell }\end{array}$ & 1.029 & DUSP5 GFI1 IRF4 TRIB1 ZBTB16 \\
\hline $\begin{array}{l}\text { Growth of } \\
\text { malignant tumor }\end{array}$ & 1.026 & $\begin{array}{l}\text { EPHA2 NR4A1 CD274 LITAF DDIT3 IRF4 } \\
\text { PLAUR PIM3 }\end{array}$ \\
\hline $\begin{array}{l}\text { Proliferation of } \\
\text { cancer cells }\end{array}$ & 1.026 & $\begin{array}{l}\text { EPHA2 NR4A1 LITAF DDIT3 IRF4 PLAUR } \\
\text { PIM3 }\end{array}$ \\
\hline $\begin{array}{l}\text { Apoptosis of } \\
\text { tumor cells }\end{array}$ & -0.728 & $\begin{array}{l}\text { NR4A1 DDIT3 IRF4 PLAUR KLF10 TRIB1 } \\
\text { PIM3 }\end{array}$ \\
\hline $\begin{array}{l}\text { Activation of } \\
\text { leukocytes }\end{array}$ & -0.758 & $\begin{array}{l}\text { ATF3 GFI1 BHLHE40 CCR7 CD274 DDIT3 } \\
\text { IRF4 AHNAK }\end{array}$ \\
\hline $\begin{array}{l}\text { Apoptosis of } \\
\text { cancer cells }\end{array}$ & -1.000 & NR4A1 DDIT3 IRF4 KLF10 TRIB1 \\
\hline
\end{tabular}

correlation between the dioxin exposure and NHL (Kogevinas et al., 1995). The increasing worldwide accidence of NHL is strongly linked to the increasing environmental pollution

Table 6 - Binding sites analysis in the promoter regions of top ten up-regulated and down-regulated genes.

\begin{tabular}{lllll} 
Gene Symbol & Gene ID & AhR & CREM & CREB1 \\
\hline Epha2 & 010139 & 2 & 2 & 2 \\
MAFF & 010755 & 1 & 6 & 1 \\
ATF3 & 007498 & 1 & 2 & 2 \\
Eps8l1 & 026146 & 0 & 2 & 0 \\
Irs2 & 001081212 & 0 & 1 & 1 \\
Osgin1 & 027950 & 0 & 1 & 0 \\
Nr4a1 & 010444 & 0 & 1 & 0 \\
Fosl2 & 008037 & 1 & 0 & 1 \\
Zbtb10 & 177660 & 0 & 2 & 0 \\
B3gnt7 & 145222 & 0 & 0 & 0 \\
Zbtb16 & 001033324 & 0 & 2 & 0 \\
Hspa1l & 013558 & 0 & 1 & 2 \\
Erdr1 & 133362 & 0 & 1 & 1 \\
Recql & 001204906 & 0 & 4 & 0 \\
Serpine2 & 009255 & 0 & 1 & 0 \\
Zbtb18 & 013915 & 1 & 1 & 0 \\
Zfp455 & 001048204 & 0 & 2 & 0 \\
Tmpo & 001080134 & 0 & 1 & 0 \\
Zfp759 & 172392 & 0 & 2 & 0 \\
Zfp948 & 001002008 & 0 & 2 & 1 \\
\hline & & & & \\
\hline & & 0 & 1 & 0 \\
\hline
\end{tabular}

(Fritschi et al., 2005). As such, NHL ranked the fifth highest diagnosed cancer in Great Britain and the incidence is increasing in many regions (Shankland et al., 2012). It is widely recognized that immunosuppression, a well-established result of TCDD exposure, is a strong risk factor for NHL, leading to 50100 fold excess risk (Evans and Hancock, 2003). However, the molecular mechanisms linking the environmental contamination to NHL are not fully elucidated. One such link can be provided by the Eph/ephrin family of the receptor tyrosine kinases known to play critical roles in lymphocyte differentiation (Cejalvo et al., 2013; Himanen et al., 2007). Upregulation of the Ephrin signaling pathway is one commonly found marker strongly associated with the oncogenic transformation (Pasquale, 2010; Xi et al., 2012). Most cases of NLH are lymphomas originated from $B$ cell at various stages of differentiation and dysregulation of normal Ephrin signaling. According to the IPA Core Analysis, the top canonical pathway induced by the chronic TCDD exposure was Ephrin A signaling pathway. Indeed, Eph receptor tyrosine kinase Ephrin A receptor proto-oncogene Eph2A was the top induced transcript in our dataset (Log2 = 10.41). Eph2A is overexpressed in most cancer types and specifically involved in B-cell leukemia (Charmsaz et al., 2015; Trinidad et al., 2009). Hypermethylation of EphA2 was detected in leukemia cell lines (Kuang et al., 2010). Because Eph2A is found to have two DREs in its promoter region, exposure to the TCDD can potentially lead to its overexpression. Moreover, because of the presence of two CREM, two CREB1 and two ATF3 binding sites in the Eph2A promoter, it is conceivable that Eph2A overexpression would be driven by a positive feedback loop realized through the cAMP signaling pathway, as mentioned above.

In addition to the extreme overexpression of the Eph2A, other proto-oncogenes including c-Maf (Log2, 2.92), Fosl2 (Log2, 2.26) and JunB (Log2, 1.03) were also highly induced. It is important to note that the c-Maf promoter was shown by CHiP analysis to be occupied by AhR (Lo and Matthews, 2012). c-Maf physically interacts with AhR to control the transcriptional activity of the IL10 promoter in Tregs (Gandhi et al., 2010); moreover, and cMaf/AhR heterodimer plays key roles in the differentiation of Th17 cells (Rutz et al., 2011). Remarkably, JunB was shown to be involved in the blockade of B cell plasmacytic differentiation (Vasanwala et al., 2002). Fosl2, ATF3 and JunB are the members of the AP1 family of transcription factor complexes, while c-Maf is a member of a family of basic leucine zipper (bZIP) proteins. Both of these protein families are prominent regulatory factors that are activated by a number of signaling cascades operating in the immune cell lineages (Ellmeier and Taniuchi, 2014). AhR is involved in the chemical sensing of the cell environment and

\begin{tabular}{|c|c|c|c|}
\hline Category & Term & $p$-Value & Genes \\
\hline KEGG_PATHWAY & $\begin{array}{l}\text { mmu04010:MAPK } \\
\text { signaling pathway }\end{array}$ & 0.014 & $\begin{array}{l}\text { DDIT3, DUSP4, } \\
\text { DUSP5, } \\
\text { HSPA1B, NR4A1 }\end{array}$ \\
\hline KEGG_PATHWAY & $\begin{array}{l}\text { mmu04710:Circadian } \\
\text { rhythm }\end{array}$ & 0.047 & $\begin{array}{l}\text { BHLHE } 40 \text {, } \\
\text { BHLHE } 41\end{array}$ \\
\hline
\end{tabular}


NHL related genes from previous report and IPA analysis

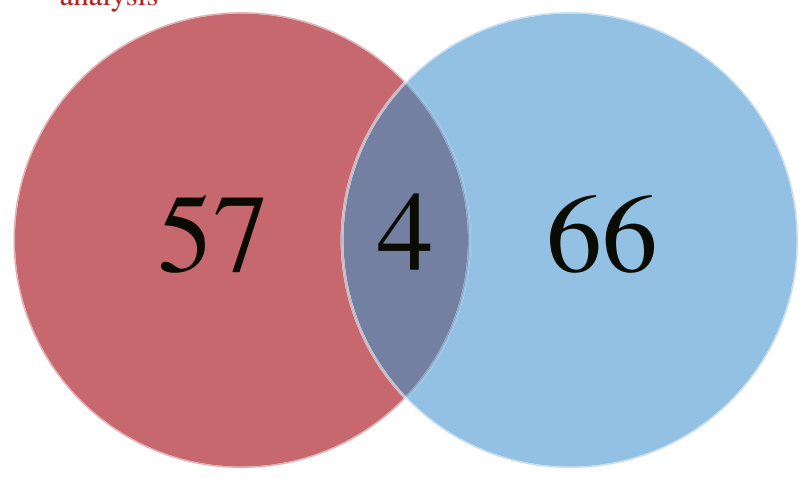

Fig. 4 - Veen diagram of NHL related genes from the previous reports and IPA analysis with the current data set showed 4 overlapping genes, IRF4, Tgif1, Fam46c and Dusp4. NHL: non-Hodgkin's lymphoma; IPA: Ingenuity Pathway Analysis.

cooperates with AP1 and bZip factors in orchestrating the cellular response (Ellmeier and Taniuchi, 2014). The most down-regulated transcript in our data set, zinc finger and BTB domain-containing protein 16 (ZBTB16) was originally identified as a fusion partner of the retinoic acid receptor $\alpha(\mathrm{RAR} \alpha)$ in acute promyeloid leukemia (Chen et al., 1993). ZBTB16 is a member of the promyelocytic leukemia zinc finger (PLZF) family of which factors are shown to be critically involved in the hematopoietic differentiation acting as sequence-specific silencers of gene expression (Siggs and Beutler, 2012). In our data set, we have 4 dysregulated members of PLZF family indicating that chronic TCDD exposure in our model was associated with profound transcriptional remodeling, consistent with the growing realization of the AhR involvement in the wide scale epigenetic remodeling (Tian, 2009). It is intriguing to think that Eph2A and ZBTB16 might be reciprocally regulated, a notion that has some support in the literature (Lan et al., 2015b).

Furthermore, the IPA core analysis showed that, following the chronic TCDD exposure, the transcripts related to apoptosis of tumor cells and cancer were down-regulated, while the transcripts involved in tumorigenesis, cell proliferation and cell invasion were up-regulated. Of note, one of the most up-regulated transcripts was an orphan nuclear receptor NR4a1. NR4a1 has been shown to play critical roles in regulation of the apoptosis (Lin et al., 2004). Moreover, NR4a1 is a critical transcriptional regulator of Foxp3 that itself is a master regulator of the development and function of Treg cells (Ellmeier and Taniuchi, 2014). While there is a consensus that TCDD exposure is a risk factor for certain cancers including NHL (Sulentic and Kaminski, 2011a), the carcinogenic potential of TCDD had been a subject of controversy (Baccarelli et al., 2004). Indeed, TCDD has both anti-inflammatory and cancer-promoting activities, and there are many factors, often counter-acting, involved in the physiology of the long term AhR activation as a result of chronic TCDD exposure, thus the adverse effects on human health can be indirect and delayed. Therefore, above results indicate that chronic TCDD exposure induces leukemogenic transcriptional program in B cells and severely affects $B$ cell differentiation. The possible mechanism underlying the change in transcriptional program can be explained using transcriptomic data.

IPA core analysis identified cAMP-dependent transcription factors CREM and CREB1 as the top upstream transcriptional regulators. The promoter analysis done with Mapper database (Marinescu et al., 2005b) showed that all but one of the most highly dysregulated genes had CREM and/or CREB1 binding sites in their promoter regions. Dysregulated pattern of gene expression associated with chronic TCDD exposure is consistent with existing evidence of the AhR involvement in chromatin remodeling (Gomez-Duran et al., 2008; Oesch-Bartlomowicz and Oesch, 2009a; Tian, 2009), potentially in concert with CREB1 in the context of cAMP/PKA signaling (Oesch-Bartlomowicz and Oesch, 2009b; Saeed et al., 2014). Involvement of cAMP/PKA signaling in the AhR signaling pathway has been demonstrated in a number of contexts including central nervous system, reproductive toxicology and wasting syndrome (Landers and Bunce, 1991; Vogel et al., 1998). The discovery we made here is a putative link between the long-term changes in cAMP signaling pathway with the AhR signaling pathway and $B$ cell differentiation. Our observations contribute to the knowledge base of molecular pathways involved in the TCDD-induced chronic immune impairment. Our data also lend support to the growing realization of the important roles of AhR signaling in normal physiology including the regulation of the circadian rhythms (Wang et al., 2014).

\section{Conclusions}

This study was designed to mimic human exposure to the persistent organic pollutant - TCDD. We used a transcriptome analysis to elucidate possible mechanisms of decreased humoral immunity and increased accidence of lymphomas characteristic of chronic human exposures to TCDD. Our murine model exhibited an overall immunosuppressive outcome from a 20-week TCDD exposure that was consistent with human exposure phenotypes. In addition, we identified a molecular signature of chronic TCDD exposure in splenic B cells that was consistent with the arrested maturation and lymphogenic transdifferentiation of these cells.

Using the IPA analysis, we identified CREM and CREB1 as the top upstream regulators of the transdifferentiation, and the ephrin signaling pathway as an effector of tumorigenic, proliferative and cell-migratory downstream effects. Ehp2A was the most up-regulated gene and thus can serve as a possible marker and/or target of the lymphogenic $B$ cell transdifferentiation. Our study has identified a putative link between CAMP and AhR signaling pathways in B cell differentiation and suggested that the cAMP signaling pathway could be an important regulator in AhR-mediated immune dysfunction. Further studies should be conducted to confirm the specific roles of the dysregulated genes in the observed pathological phenotype, including the specific molecular mechanisms involved in the arrested B cell maturation. 


\section{Acknowledgment}

This work was supported by the National Natural Science Foundation of China (No. 21277168, 21525730), the Strategic Priority Research Program of the Chinese Academy of Sciences (Nos. XDB14030401, XDB14030402), and Chinese Academy of Sciences President's International Fellowship to Irina Krylova (No. 2015VBC063). We thank Dr. Marjorie A. Phillips from UC Davis for discussion and comments during the manuscript preparation.

\section{Appendix A. Supplementary data}

Supplementary data to this article can be found online at http://dx.doi.org/10.1016/j.jes.2015.11.011.

\section{REFEREN C ES}

Ashburner, M., Ball, C.A., Blake, J.A., Botstein, D., Butler, H., Cherry, J.M., et al., 2000. Gene ontology: tool for the unification of biology. Nat. Genet. 25 (1), 25-29.

Baccarelli, A., Pesatori, A., Masten, S., Patterson, D., Needham, L., Mocarelli, P., et al., 2004. Aryl-hydrocarbon receptor-dependent pathway and toxic effects of TCDD in humans: a population-based study in Seveso. Italy. Toxicol. Lett. 149 (1-3), 287-293.

Carvajal-Gonzalez, J., Mulero-Navarro, S., Roman, A., Sauzeau, V., Merino, J., Bustelo, X., et al., 2009. The dioxin receptor regulates the constitutive expression of the vav3 proto-oncogene and modulates cell shape and adhesion. Mol. Biol. Cell 20 (6), 1715-1727.

Cejalvo, T., Munoz, J., Tobajas, E., Fanlo, L., Alfaro, D., GarcíaCeca, J., et al., 2013. Ephrin-B-dependent thymic epithelial cell-thymocyte interactions are necessary for correct $\mathrm{T}$ cell differentiation and thymus histology organization: relevance for thymic cortex development. J. Immunol. 190 (6), 2670-2681.

Charmsaz, S., Beckett, K., Smith, F., Bruedigam, C., Moore, A., Al-Ejeh, F., et al., 2015. EphA2 is a therapy target in EphA2-positive leukemias but is not essential for normal hematopoiesis or leukemia. PloS one 10 (6), e0130692.

Chen, Z., Brand, N., Chen, A., Chen, S., Tong, J., Wang, Z., et al., 1993. Fusion between a novel Krüppel-like zinc finger gene and the retinoic acid receptor-alpha locus due to a variant $t(11 ; 17)$ translocation associated with acute promyelocytic leukaemia. EMBO J. 12 (3), 1161-1167.

De Abrew, K., Kaminski, N., Thomas, R., 2010. An integrated genomic analysis of aryl hydrocarbon receptor-mediated inhibition of B-cell differentiation. Toxicol. Sci. 118 (2), 454-469.

De Abrew, K., Phadnis, A., Crawford, R., Kaminski, N., Thomas, R., 2011. Regulation of Bach2 by the aryl hydrocarbon receptor as a mechanism for suppression of B-cell differentiation by 2,3,7,8-tetrachlorodibenzo-p-dioxin. Toxicol. Appl. Pharmacol. 252 (2), 150-158.

Dennis Jr., G., Sherman, B.T., Hosack, D.A., Yang, J., Gao, W., Lane, H.C., et al., 2003. DAVID: database for annotation, visualization, and integrated discovery. Genome. Biol. 4 (5), P3.

DeVito., M.J., Birnbaum, L.S., Farland, W.H., Gasiewicz, T./.A., 1995. Comparisons of estimated human body burdens of dioxinlike chemicals and TCDD body burdens in experimentally exposed animals. Environ. Health. Persp. 103 (9), 820-831.
Dooley, R.K., Holsapple, M.P., 1988. Elucidation of cellular targets responsible for tetrachlorodibenzo-p-dioxin (TCDD)-induced suppression of antibody responses: I. The role of the $B$ lymphocyte. Immunopharmacology 16 (3), 167-180.

Ellmeier, W., Taniuchi, I., 2014. Transcriptional control of lineage differentiation in immune cells. Springer 21-51.

Evans, L.S., Hancock, B.W., 2003. Non-hodgkin lymphoma. Lancet 362 (9378), 139-146.

Fernandez-Salguero, P., 2010. A remarkable new target gene for the dioxin receptor: the Vav3 proto-oncogene links AhR to adhesion and migration. Cell. Adh. Migr. 4 (2), 172-175.

Fritschi, L., Benke, G., Hughes, A., Kricker, A., Vajdic, C., Grulich, A., et al., 2005. Risk of non-Hodgkin lymphoma associated with occupational exposure to solvents, metals, organic dusts and PCBs (Australia). Cancer Causes Control 16 (5), 599-607.

Gandhi, R., Kumar, D., Burns, E., Nadeau, M., Dake, B., Laroni, A., et al., 2010. Activation of the aryl hydrocarbon receptor induces human type 1 regulatory T cell-like and Foxp3(+) regulatory T cells. Nat. Immunol. 11 (9), 846-853.

Gomez-Duran, A., Ballestar, E., Carvajal-Gonzalez, J., Marlowe, J., Puga, A., Esteller, M., et al., 2008. Recruitment of CREB1 and histone deacetylase 2 (HDAC2) to the mouse Ltbp-1 promoter regulates its constitutive expression in a dioxin receptor-dependent manner. J. Mol. Biol. 380 (1), 1-16.

Gomez-Duran, A., Carvajal-Gonzalez, J., Mulero-Navarro, S., Santiago-Josefat, B., Puga, A., Fernandez-Salguero, P., 2009. Fitting a xenobiotic receptor into cell homeostasis: how the dioxin receptor interacts with TGFbeta signaling. Biochem. Pharmacol. 77 (4), 700-712.

Himanen, J.P., Saha, N., Nikolov, D.B., 2007. Cell-cell signaling via Eph receptors and ephrins. Curr. Opin. Cell. Biol. 19 (5), 534-542.

Ho, P.P., Steinman, L., 2008. The aryl hydrocarbon receptor: a regulator of Th17 and Treg cell development in disease. Cell. Res. 18 (6), 605-608.

Holsapple, M.P., Morris, D.L., Wood, S.C., Snyder, N.K., 1991. 2,3,7,8-Tetrachlorodibenzo-p-dioxin-induced changes in immunocompetence: possible mechanisms. Annu. Rev. Pharmacol. 31 (1), 73-100.

Huang, D.W., Sherman, B.T., Zheng, X., Yang, J., Imamichi, T., Stephens, R., et al., 2009. Extracting biological meaning from large gene lists with DAVID. Curr. Protoc. Bioinformatics (Chapter 13:Unit 13.11).

Kanehisa, M., Goto, S., 2000. KEGG: kyoto encyclopedia of genes and genomes. Nucleic. Acids. Res. 28 (1), 27-30.

Kerkvliet, N., 2009a. AHR-mediated immunomodulation: the role of altered gene transcription. Biochem. Pharmacol. 77 (4), 746-760.

Kerkvliet, N.I., 2002. Recent advances in understanding the mechanisms of TCDD immunotoxicity. Int. Immunopharmacol. 2 (2-3), 277-291.

Kerkvliet, N.I., 2009b. AHR-mediated immunomodulation: the role of altered gene transcription. Biochem. Pharmacol. 77 (4), 746-760.

Kidd, P., 2003. Th1/Th2 balance: the hypothesis, its limitations, and implications for health and disease. AlternL. Med. Rev. : a journal of clinical therapeutic 8 (3), 223-246.

Kogevinas, M., Kauppinen, T., Winkelmann, R., Becher, H., Bertazzi, P.A., Bueno-de-Mesquita, H.B., et al., 1995. Soft tissue sarcoma and non-Hodgkin's lymphoma in workers exposed to phenoxy herbicides, chlorophenols, and dioxins: two nested case-control studies. Epidemiology 6 (4), 396-402.

Krämer, A., Green, J., Pollard, J., Tugendreich, S., 2014. Causal analysis approaches in Ingenuity Pathway Analysis. Bioinformatics 30 (4), 523-530.

Kuang, S., Bai, H., Fang, Z., Lopez, G., Yang, H., Tong, W., et al., 2010. Aberrant DNA methylation and epigenetic inactivation of Eph receptor tyrosine kinases and ephrin ligands in acute lymphoblastic leukemia. Blood 115 (12), 2412-2419. 
Lan, X., Zhang, H., Wang, Z., Don, W., Sun, W., Shao, L., et al., 2015a. Genome-wide analysis of long noncoding RNA expression profile in papillary thyroid carcinoma. Gene 569 (1), 109-117.

Lan, X., Zhang, H., Wang, Z., Dong, W., Sun, W., Shao, L., et al., $2015 \mathrm{~b}$. Genome-wide analysis of long noncoding RNA expression profile in papillary thyroid carcinoma. Gene 569 (1), 109-117.

Landers, J.P., Bunce, N.J., 1991. The Ah receptor and the mechanism of dioxin toxicity. Biochem. J. 276 (Pt 2), 273.

Li, Y., Innocentin, S., Withers, D.R., Roberts, N.A., Gallagher, A.R., et al., 2011. Exogenous stimuli maintain intraepithelial lymphocytes via aryl hydrocarbon receptor activation. Cell 147 (3), 629-640

Li, Z., Xu, H., Zheng, W., Lam, S., Gong, Z., 2013. RNA-sequencing analysis of TCDD-induced responses in zebrafish liver reveals high relatedness to in vivo mammalian models and conserved biological pathways. PloS one 8 (10), e77292.

Licona-Limón, P., Kim, L.K., Palm, N.W., Flavell, R.A., 2013. TH2, allergy and group 2 innate lymphoid cells. Nat. Immunol. 14 (6), 536-542

Lin, B., Kolluri, S., Lin, F., Liu, W., Han, Y., Cao, X., et al., 2004. Conversion of $\mathrm{Bcl}-2$ from protector to killer by interaction with nuclear orphan receptor Nur77/TR3. Cell 116 (4), 527-540.

Lo, R., Matthews, J., 2012. High-resolution genome-wide mapping of AHR and ARNT binding sites by ChIP-Seq. Toxicol. Sci. 130 (2), 349-361

Lund, A., Goens, M., Kanagy, N., Walker, M., 2003. Cardiac hypertrophy in aryl hydrocarbon receptor null mice is correlated with elevated angiotensin II, endothelin-1, and mean arterial blood pressure. Toxicol. Appl. Pharmacol. 193 (2), 177-187.

MacLeod, M., David, A., McKee, A., Crawford, F., Kappler, J., Marrack, P., 2011. Memory CD4 T cells that express CXCR5 provide accelerated help to B cells. J. Immunol. 186 (5), 2889-2896.

Marinescu, V., Kohane, I., Riva, A., 2005a. The MAPPER database: a multi-genome catalog of putative transcription factor binding sites. Nucleic Acids Res. 33 (Database issue), D91-D97.

Marinescu, V., Kohane, I., Riva, A., 2005b. MAPPER: a search engine for the computational identification of putative transcription factor binding sites in multiple genomes. BMC Bioinformatics 6, 79.

Mortazavi, A., Williams, B.A., McCue, K., Schaeffer, L., Wold, B., 2008. Mapping and quantifying mammalian transcriptomes by RNA-Seq. Nat. methods 5 (7), 621-628.

Negishi, T., Kato, Y., Ooneda, O., Mimura, J., Takada, T., Mochizuki, H., et al., 2005. Effects of aryl hydrocarbon receptor signaling on the modulation of TH1/TH2 balance. J. Immunol. 175 (11), 7348-7356.

Nguyen, N.T., Hanieh, H., Nakahama, T., Kishimoto, T., 2013. The roles of aryl hydrocarbon receptor in immune responses. Int. Immunol. 25 (6), 335-343.

Oesch-Bartlomowicz, B., Oesch, F., 2009a. Role of cAMP in mediating AHR signaling. Biochem. Pharmacol. 77 (4), 627-641.

Oesch-Bartlomowicz, B., Oesch, F., 2009b. Role of cAMP in mediating AHR signaling. Biochem. Pharmarcol. 77 (4), 627-641.

Oughton, J.A., Pereira, C.B., DeKrey, G.K., Collier, J.M., Frank, A.A., et al., 1995. Phenotypic analysis of spleen, thymus, and peripheral blood cells in aged C57B1/6 mice following long-term exposure to 2,3,7,8-tetrachlorodibenzo-p-dioxin. Fundam. Appl. Toxicol. 25 (1), 60-69.

Pasquale, E., 2010. Eph receptors and ephrins in cancer: bidirectional signalling and beyond. Nat. Rev. Cancer 10 (3), 165-180.

Rutz, S., Noubade, R., Eidenschenk, C., Ota, N., Zeng, W., Zheng, Y., et al., 2011. Transcription factor c-Maf mediates the TGF- $\beta$-dependent suppression of IL-22 production in T(H)17 cells. Nat. Immunol. 12 (12), 1238-1245.

Saeed, S., Quintin, J., Kerstens, H., Rao, N., Aghajanirefah, A., Matarese, F., et al., 2014. Epigenetic programming of monocyte-to-macrophage differentiation and trained innate immunity. Science 345 (6204), 1251086.
Santiago-Josefat, B., Mulero-Navarro, S., Dallas, S., Fernandez-Salguero, P., 2004. Overexpression of latent transforming growth factor-beta binding protein 1 (LTBP-1) in dioxin receptor-null mouse embryo fibroblasts. J. Cell. Sci. 117 (Pt 6), 849-859.

Schulz, V.J., Smit, J.J., Willemsen, K.J., Fiechter, D., Hassing, I., et al., 2011. Activation of the aryl hydrocarbon receptor suppresses sensitization in a mouse peanut allergy model. Toxicol. Sci. 123 (2), 491-500.

Shankland, K.R., Armitage, J.O., Hancock, B.W., 2012. Non-Hodgkin lymphoma. The Lancet 380 (9844), 848-857.

Siggs, O., Beutler, B., 2012. The BTB-ZF transcription factors. Cell Cycle 11 (18), 3358-3369.

Smith, K., Pottage, L., Thomas, E., Leishman, A., Doig, T., Xu, D., et al., 2000. Th1 and Th2 CD4 + T cells provide help for B cell clonal expansion and antibody synthesis in a similar manner in vivo. J. Immunol. 165 (6), 3136-3144.

Spits, H., Di Santo, J.P., 2011. The expanding family of innate lymphoid cells: regulators and effectors of immunity and tissue remodeling. Nat. Immunol. 12 (1), 21-27.

Stubbington, M., Mahata, B., Svensson, V., Deonarine, A., Nissen, J., Betz, A., et al., 2015. An atlas of mouse CD4(+) T cell transcriptomes. Biol. Direct. 10 (1), 10-14.

Sulentic, C., Kaminski, N., 2011a. The long winding road toward understanding the molecular mechanisms for B-cell suppression by 2,3,7,8-tetrachlorodibenzo-p-dioxin. Toxicol. Sci. 120 (Suppl. 1), S171-S191.

Sulentic, C.E., Kaminski, N.E., 2011b. The long winding road toward understanding the molecular mechanisms for B-cell suppression by 2,3,7,8-tetrachlorodibenzo-p-dioxin. Toxicol. Sci. 120 (Suppl. 1), S171-S191.

Tian, Y., 2009. Ah receptor and NF-kappaB interplay on the stage of epigenome. Biochem. Pharmacol. 77 (4), 670-680.

Trinidad, E., Ballesteros, M., Zuloaga, J., Zapata, A., Alonso-Colmenar, L., 2009. An impaired transendothelial migration potential of chronic lymphocytic leukemia (CLL) cells can be linked to ephrin-A4 expression. Blood 114 (24), 5081-5090.

Vasanwala, F., Kusam, S., Toney, L., Dent, A., 2002. Repression of AP-1 function: a mechanism for the regulation of Blimp-1 expression and B lymphocyte differentiation by the B cell lymphoma-6 protooncogene. J. Immunol. 169 (4), 1922-1929.

Vogel, C., Schuhmacher, U.S., Degen, G.H., Bolt, H.M., Pineau, T., Abel, J., 1998. Modulation of prostaglandin H synthase- 2 mRNA expression by 2, 3, 7, 8-tetrachlorodibenzo-p-dioxin in mice. Arch. Biochem. Biophys. 351 (2), 265-271.

Vorderstrasse, B.A., Steppan, L.B., Silverstone, A.E., Kerkvliet, N.I., 2001a. Aryl hydrocarbon receptor-deficient mice generate normal immune responses to model antigens and are resistant to TCDD-induced immune suppression. Toxicol. Appl. Pharmacol. 171 (3), 157-164.

Vorderstrasse, B.A., Steppan, L.B., Silverstone, A.E., Kerkvliet, N.I., 2001b. Aryl hydrocarbon receptor-deficient mice generate normal immune responses to model antigens and are resistant to TCDD-induced immune suppression. Toxicol. Appl. Pharmacol. 171 (3), 157-164.

Wang, C., Zhang, Z., Xu, C., Tischkau, S., 2014. Interplay between Dioxin-mediated signaling and circadian clock: a possible determinant in metabolic homeostasis. Int. J. Mol. Sci. 15 (7), 11700-11712.

Xi, H., Wu, X., Wei, B., Chen, L., 2012. Eph receptors and ephrins as targets for cancer therapy. J. Cell. Mol. Med. 16 (12), 2894-2909.

Zhang, Q., Kline, D., Bhattacharya, S., Crawford, R., Conolly, R., Thomas, R., et al., 2013. All-or-none suppression of B cell terminal differentiation by environmental contaminant 2,3,7,8-tetrachlorodibenzo-p-dioxin. Toxicol. Appl. Pharmacol. 268 (1), 17-26. 